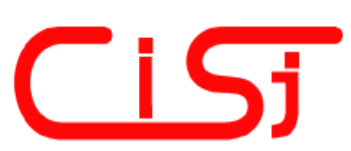

\title{
SYNTHESIS OF THE REGULATOR FOR ROBOT'S ARM SERVING BY POLES METHOD PLACEMENT USING
}

\author{
Markiyan Nakonechnyi ${ }^{1)}$, Orest Ivakhiv ${ }^{2)}$, Oleksandr Viter ${ }^{1)}$ \\ 1) Department of Computerized Automation Systems, Lviv Polytechnic National University \\ 12 Bandera str., Lviv 79013 Ukraine, viteros45@ gmail.com \\ ${ }^{2)}$ Precision Mechanics Department, Lviv Polytechnic National University \\ 12 Bandera str., Lviv 79013 Ukraine, oresti @ polynet.lviv.ua
}

Paper history:

Received 15 March 2019

Received in revised form 7 February 2020

Accepted 10 February 2020

Available online 14 June 2020

Keywords:

synthesis;

controller;

robot;

servo drive;

poles;

state variables;

MATLAB;

Simulink.

\begin{abstract}
This paper presents the synthesis of a controller for robot arm servo drive by placing the poles of the transfer function. The problem of synthesis is determined, based on the desired duration of the transition process, the desired location of the roots of the characteristic equation of closed system and space state variables regulator factors are found that provide the desired system performance. There was used Ackerman's formula for the synthesis of regulator that allows for the placement of all poles of the transfer function of a closed system at given points of the plane of the roots of the characteristic equation. Synthesis by placing poles is based on the use of model automatic control system state variables in space. As well as there is synthesized block diagram of the controller for robot arm servo drive and both held it modeling in MATLAB and moved it in Simulink environment. Due to the stated values of transition time and output signal displacement that provide the allowed divergence between output signal and sample, proposed control system works in automatic mode.

Copyright C Research Institute for Intelligent Computer Systems, 2020.
\end{abstract} All rights reserved.

\section{INTRODUCTION}

The task of creating any system of automatic control is to complement the controlled object with such external links that ensure the passage of processes in an object in accordance with certain preformulated criteria.

The choice of these criteria is determined by the fact that the purpose of the functioning of the automatic control system is to provide at any time at the output of the controlled object such regulated value that is the closest to the given one. If this value is constant, then the system will be a system of stabilization, but when it changes over time in advance of a known law, then we are dealing with the system of software control. In cases where the law of change of the target value is unknown, one needs the tracking system using. Consequently, the criterion of the quality of the functioning of the system should reflect the degree of approaching the output signals to the given, taking into account the need to comply with certain additional requirements.
Any real controlled dynamic object is characterized by certain features, the main of which is its inertia, when changing the input value, it has a transitional process. In addition, in some cases, the object is under the various perturbations, which leads to a change in the values of the original value at a constant value of the input. The system should function correctly, taking into account the effect of these factors.

As it is known, the methods of synthesis of regulators, which today are used in the design of linear systems of automatic control, are based on the use of frequency characteristics or the root hodograph. These methods are convenient in practical use and most control systems are designed based on their various modifications.

For example, the method of a root hodograph involves the synthesis of a regulator by placing in a given region of the dominant roots of the characteristic equation of the system, which is formed on the basis of the regulator and represented in the form of the transfer function of the object 
model. The frequency response method uses the frequency characteristics of the controller and the object. In both cases, the requirements for the formed system are given indirectly - either in the form of a given zone of the arrangement of the roots of the characteristic equation, or in the form of the desired frequency characteristic [1-7].

Concerning the nonlinear dynamical systems, it is difficult to construct an analytical model in this case, so it is expedient to use a method that provides the capability of automatically building up the necessary models. This method is based on using artificial neural network [8-16] as a universal approximating device, which in the process of training based on defined sequences, can accommodate to the input data in order to get at the output of the network the values as close as possible to the related output signals given in the form of the objective function.

\section{TASK SOLUTION}

The traditional classical method of studying the dynamics of automatic control systems is that the equations of the links of the system form a general structural diagram of the system and determine the overall transfer function of a closed system, which connects the input and output quantities. One general differential equation of the $n$-th order closed system can be obtained from the transfer function. However, the behavior of the system over time can be characterized not only by the initial value of the system, but also by the intermediate variables in the system chain, whose number is equal to the order of the system $n$. These intermediate variables should be chosen so that after setting the initial values, these $n$ variables could determine the value of the output variable at any subsequent point in time, that is, determine the state of the system at any point in time. This $n$-dimensional vector of variables is called the state vector of the system, the whole set of possible values of this vector is the space of states of the system [3]. In the space of states, the properties of the control system are described not by one $n$ order differential equation but by a system of $n$ firstorder differential equations in the normal form. One can choose the state variables and go to the system of equations in the normal form in different ways.

In recent years, considerable attention has been paid to the development of new methods for the synthesis of regulators that allow the placement of all poles of the transfer function of a closed system at given points of the plane of the roots of the characteristic equation. The synthesis of regulators using such methods involves the construction of a mathematical model of an object in the space of state variables, and the structural scheme of a closed system is presented in the form shown in Fig. 1.

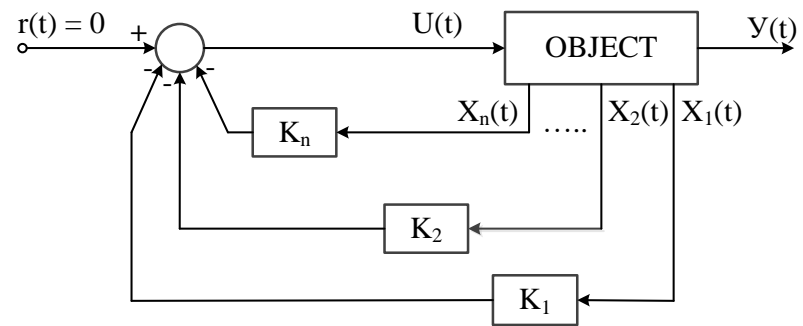

Figure 1 - Structural scheme of the system based on the location of the poles

Since in our case the zero signal is fed to the system's input, its purpose is to maintain the original output equal to zero. In real conditions, the system is affected by external perturbations, the effect of which leads to a deviation of the output value from zero. The purpose of feedback is the return of the output value and all the variables of the state to zero by the corresponding influences whose action is formed by a synthesized regulator based on the chosen control law.

Consider a synthesis method known as the placement method or the pole assignment. This method is similar to a root hodograph synthesis method, the use of which allows us to place the individual poles of the closed-loop transfer function at predetermined points. The method using modern control theory [2] allows us to realize the given position of all poles of the closed-loop transfer function at given points, whereas the root-hodograph method provides for the placement of only two dominant poles at given points. The peculiarity of the method is that the placement of all poles of the transfer function at a given point requires the measurement of all the variables that characterize the flow of the process in the object. In practice, due to the complexity or lack of suitable converters, not all the variables necessary for the synthesis of the controller can be measured. In such cases, those variables that cannot be measured directly are subject to evaluation on the basis of the resulting measurement variables.

Mathematical models in the form of object transfer functions are used in the classical synthesis of regulators synthesis procedures. Synthesis of the controller by positioning the poles involves the use of the object model in the space of state variables. A synthesis procedure for a controller based on the location of the poles of the closed-loop transfer function at given points is considered in [3].

The features of the synthesis of the regulator for a linear stationary system whose equation of object in the space of the state variables has the form as follows: 


$$
\begin{gathered}
\dot{x}(t)=A x(t)+B u(t), \\
y(t)=C x(t),
\end{gathered}
$$

where the vector $\dot{x}(t)$ is a time derivative of the vector of the state $x(t)$ with dimension $(n \times 1)$; its components are the $n$-order object state variables; $A$ is a matrix of coefficients with dimension $(n \times n) ; B$ is a matrix of coefficients with dimension $(n \times r)$; $u(t)$ is the input vector with dimension $(r \times 1)$, the components of which are input object variables; $y(t)$ is the output vector with dimension $(p \times 1)$, the components of which are the output object variables; $C$ is the output matrix with dimension $(p \times n)$.

The control law is determined by the equation as follows:

$$
u(t)=f[x(t)]
$$

In the synthesis of the regulator by the method of placement of the poles at given points of the plane of the roots, the law of control is given in the following form:

$$
u(t)=-K x(t)
$$

Substituting (4) into (1), we obtain as follows:

$$
\dot{x}(t)=A x(t)-B K x(t)=(A-B K) \cdot x(t)=A_{f} x(t),
$$

where $A_{f}=(A-B K)$ is the matrix of coefficients of a closed system.

The characteristic equation of a closed system is written as follows:

$$
\left|s I-A_{f}\right|=|s I-A+B K|=0,
$$

where $s$ is the Laplace operator and $I$ is the unit matrix.

Assume that under the conditions of synthesis, the roots of the characteristic equation of the system should be equal to $-\lambda_{1},-\lambda_{2}, \ldots,-\lambda_{n}$. The desired characteristic equation of the system is written as follows:

$$
\begin{aligned}
& \alpha_{c}(s)=s^{n}+a_{n-1} s^{n-1}+\ldots+a_{1} s+a_{0}= \\
& =\left(s+\lambda_{1}\right)\left(s+\lambda_{2}\right) \ldots\left(s+\lambda_{n}\right)=0
\end{aligned}
$$

In accordance with the procedure of synthesis by placing poles, it is necessary to find a matrix such as that the expressions (6) and (7) become equal. That is as follows

$$
|s I-A+B K|=s^{n}+\alpha_{n-1} s^{n-1}+\ldots+\alpha_{1} s+\alpha_{0} .
$$

The equation (8) contains $n$ unknowns $\left(K_{1}, K_{2}, \ldots, K_{n}\right)$. Equating in this equation coefficients at the same degrees $s$, it is obtained $n$ linear algebraic equations for $n$ unknowns. Having solved these equations, one finds the elements of the matrix $K$.

Ackerman [17] proposed a formula for calculating the elements of the matrix $K$, which satisfies the equation (8). This formula is based on the transformation of similarity, which translates a given model of an arbitrary structure into a canonical form of controllability, and then, using (7) and (8), unknown coefficients of $K_{i}$ are determined. Then the resulting solution is recalculated in relation to the original structure. These actions are performed using the following Ackerman formula:

$$
K=[00 \ldots 01]\left[B A B \ldots A^{n-2} B A^{n-1} B\right] \alpha_{c}(A),
$$

where $\alpha_{c}(A)$ is a matrix polynomial formed by using the coefficients of the desired characteristic equation with $\alpha_{c}(s)$, that is,

$$
a_{c}(A)=A^{n}+a_{n-1} A^{n-1}+\ldots+a_{1} A+a_{0} I .
$$

Most industrial robots have hydraulic, electrical or pneumatic actuators. Electric actuators are often equipped with DC motors with independent excitation at each link. As a rule, DC motors are motors with permanent magnet, anchor excitation and continuous rotation of the output shaft. They are characterized by high power, smooth running, the ability to work at low speeds, the linearity of the load characteristics and a small time constant. The use of constant magnetic field and direct current energy provides maximum momentum with minimal energy consumed for power and minimal weight. These parameters also reduce the inductance of the motor and, accordingly, the steady-state value caused by electrical interactions. So, let us consider the method of a single link robot the transfer function obtaining, which can be used in the synthesis of proportional-integral controller. In the analysis, one unit of work is treated as a linear continuous system to which the Laplace transform method can be used to simplify the calculations. It was taken a DC motor with control in the anchor link circuit.

The load link (robot arm) is connected to the output shaft of the engine by a gearbox (Fig. 2), forming a mechanical transmission system [20].

The gear ratio $n$ is proportional to the ratio of the teeth (radii) of the interacting gears of the inner and outer gearboxes, and since the joint movement of the gearboxes in their interaction is the same. 


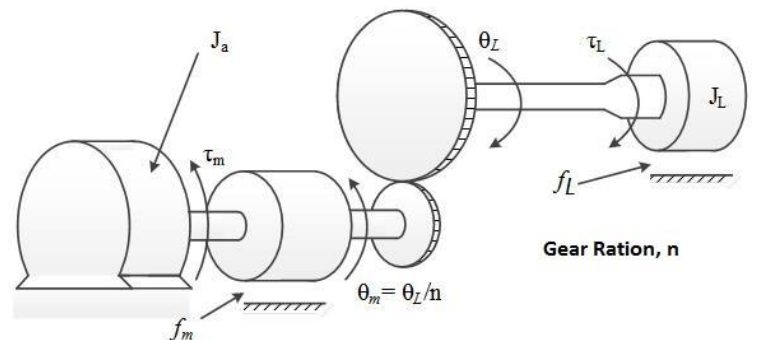

Figure 2 - The scheme of a torque from the motor to loading transmission

Hence the relationship between the angular displacements (in radians) of the motor shaft $\theta_{\mathrm{m}}$ and the load shaft $\theta_{\mathrm{L}}$ is as follows:

$$
\theta_{L}(t)=n \theta_{m}(t)
$$

After double differentiation of expression (11) one obtains the following:

$$
\begin{gathered}
\dot{\theta}_{L}(t)=n \dot{\theta}_{m}(t) \\
\ddot{\theta}_{L}(t)=n \ddot{\theta}_{m}(t) .
\end{gathered}
$$

The motor torque on its shaft is equal to

$$
\tau_{m}(t)=J_{m} \ddot{\theta}_{m}(t)+f_{m} \dot{\theta}_{m}(t)
$$

and the load moment reduced to the load shaft is equal to

$$
\tau_{L}(t)=J_{L} \ddot{\theta}_{L}(t)+f_{L} \dot{\theta}_{L}(t)
$$

where, $J_{m}$ and $J_{L}$ is the moment of inertia $\left(\mathrm{N} \mathrm{m} \mathrm{s}^{2} /\right.$ rad) of the motor reduced to the motor shaft and the load reduced to the load shaft, respectively; $f_{m}$ and $f_{L}$ is the coefficient of viscous friction $(\mathrm{N} \mathrm{m} \mathrm{s} / \mathrm{rad})$ of the motor, reduced to the motor shaft and the load, respectively, reduced to the load shaft.

According to the law of the energy conservation, the work performed by the load reduced to the shaft equal to $\tau_{\mathrm{L}} \theta_{\mathrm{L}}$ must be the same as the work reduced to the motor shaft equal to $\tau_{\mathrm{L}}{ }^{*} \theta_{\mathrm{m}}$. On this basis, one obtains as follows:

$$
\tau_{L}^{*}(t)=\frac{\tau_{L}(t) \theta_{L}(t)}{\theta_{m}(t)}=n \tau_{L}(t)
$$

Given equations (12), (13), and (15) one obtains as follows:

$$
\tau_{L}^{*}(t)=n^{2}\left[J_{L} \ddot{\theta}_{m}(t)+f_{L} \dot{\theta}(t)\right] .
$$

Since the load is connected to an external gearbox, the moment created at the output shaft of the motor is equal to the sum of the moments of the motor $\tau_{m}(t)$ and the load reduced to the motor shaft is as follows:

$$
\tau(t)=\tau_{m}(t)+\tau_{L}^{*}(t)
$$

Using equations (14) and (17), the expression for the moment created at the output shaft of the motor is as follows:

$$
\begin{aligned}
& \tau(t)=\tau_{m}(t)+\tau_{L}^{*}(t)=\left(J_{m}+n^{2} J_{L}\right) \ddot{\theta}_{m}(t)+ \\
& +\left(f_{m}+n^{2} f_{L}\right) \dot{\theta}_{m}(t)=J_{e f f} \ddot{\theta}_{m}(t)+f_{e f f} \dot{\theta}_{m}(t)
\end{aligned}
$$

where $J_{\text {eff }}=J_{m}+n^{2} J_{L}$ and $f_{\text {eff }}=f_{m}+n^{2} f_{L}$ is the total effective moment of inertia of the motor and load and the total coefficient of friction of motor viscosity and load, respectively, reduced to the shaft of the motor.

Based on the above results, it is possible [20] to determine the transfer function of a single link system (Fig. 3).

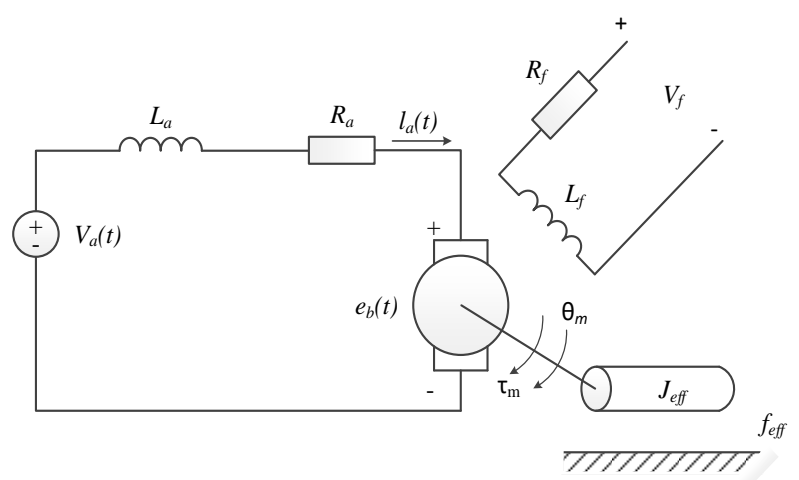

Figure 3 - Equivalent circuit of an electric drive with a DC motor and a control in the anchor circle

Since the torque on the motor shaft is linearly dependent on the anchor current and independent on speed and angular position, one obtains the following:

$$
\tau(t)=K_{a} i_{a}(t),
$$

where $K_{a}$ is the coefficient of proportionality having the dimension $[\mathrm{N} \mathrm{m} / \mathrm{A}]$.

Using Kirchhoff's law for the anchor circuit, one obtains the following:

$$
\begin{gathered}
V_{a}(t)=R_{a} i_{a}(t)+L_{a} \frac{d i_{a}(t)}{d t}+e_{b}(t), \\
\mathrm{e}_{\mathrm{b}}(\mathrm{t})=(t)=K_{b} \dot{\theta}_{m}(t),
\end{gathered}
$$

where $e_{b}$ is the electromotive force proportional to the angular velocity of the motor and $K_{b}$ is a coefficient of proportionality having the dimension [V s / rad]. 
Performing the Laplace transform over the obtained equations and solving them with respect to $I_{a}(s)$, one obtains as follows:

$$
\mathrm{I}_{\mathrm{a}}(\mathrm{s})=\frac{\mathrm{V}_{\mathrm{a}}(\mathrm{s})-\mathrm{s} \mathrm{K}_{\mathrm{b}} \theta_{\mathrm{m}}(s)}{R_{a}+s L_{a}} .
$$

As a result of the Laplace transform above equation (17) one obtains as follows:

$$
T(s)=s^{2} J_{e f f} \theta_{m}(s)+s f_{e f f} \theta_{m}(s) .
$$

Performing the Laplace transform over equation (20) and substituting the value $I_{a}(s)$ from equation (23), one obtains as follows:

$$
T(s)=K_{a} I_{a}(s)=K_{a}\left[\frac{V_{a}(s)-s K_{b} \theta_{m}(s)}{R_{a}+s L_{a}}\right]
$$

Equating (24) and (25) and grouping the corresponding terms, the transfer function from the anchor voltage to the angular displacement of the motor shaft is written in the following form:

$$
\frac{\theta_{m}(s)}{V_{a}(s)}=\frac{K_{a}}{s\left[s^{2} J_{e f f}+\left(L_{a} f_{e f f}+R_{a} J_{e f f}\right) s+R_{a} f_{e f f}+K_{a} K_{b}\right]}(26)
$$

The block diagram (Fig. 2) is prepared according to the transfer function (26).

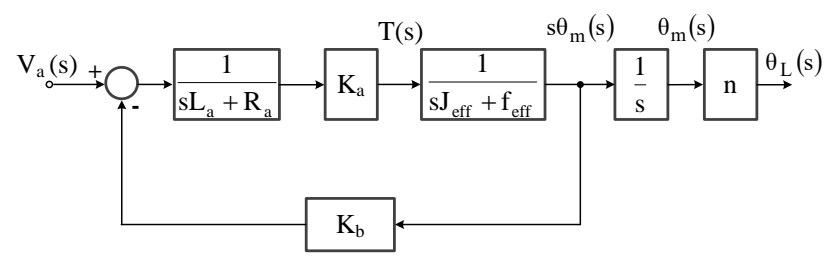

Figure 4 - Structural diagram of one robot link after the angle

To synthesize the controller, it is necessary to set the numerical values of the parameters of one link of the robot [18]. Namely, motor winding resistance $R a=0.2[\mathrm{Ohm}]$; the inductance of the winding of the motor $\mathrm{La}-0.02[\mathrm{Hn}]$; coefficient of gear ratio $n=0.1$; motor inertia moment $\mathrm{Jm}=1.3 * 10^{-6}$ $\left[\mathrm{kg} * \mathrm{~m}^{2}\right]$; loading inertia moment $\mathrm{Jl}=0.6 * 10^{-3}$ $\left[\mathrm{kg} * \mathrm{~m}^{2}\right]$; the friction coefficient of the motor $F m=0[\mathrm{~N} * \mathrm{~m} * \mathrm{~s}] ;$ the viscous friction coefficient of the load $\mathrm{Fl}=0.53 * 10^{-2}[\mathrm{~N} * \mathrm{~m} * \mathrm{~s}]$; coefficient of the motor moment transmission $\mathrm{Ka}=0.8 * 10^{-5}$ $[\mathrm{H} * \mathrm{~m} / \mathrm{A}]$; coefficient of the opposite e.m.f. $K b=5.5 * 10^{-2}[\mathrm{~V} * \mathrm{~s} / \mathrm{rad}]$.
Because of the calculations carried out, the transmitting function of one link after the angle (26) in numerical form is as follows:

$$
W_{\theta}(s)=\frac{54.79}{s^{3}+17.26 s^{2}+75.62 s}=\frac{\theta(s)}{V(s)} .
$$

The mathematical model of robot link after the angle in the space of variables that corresponds to expression (27) has the following form:

$$
\begin{gathered}
\dot{x}(t)=A x(t)+B u(t) \text { and } y(t)=C x(t) ; \\
A=\left|\begin{array}{ccc}
-17.26 & -75.62 & 0 \\
1 & 0 & 0 \\
0 & 1 & 0
\end{array}\right| ; \\
B=\left|\begin{array}{c}
1 \\
0 \\
0
\end{array}\right| ; \\
C=\left|\begin{array}{lll}
0 & 0 & 54.79
\end{array}\right| .
\end{gathered}
$$

The block diagram of the system operation is formed (Fig. 5) when the angle in the space of the variables corresponds to the relation (28) - (31).

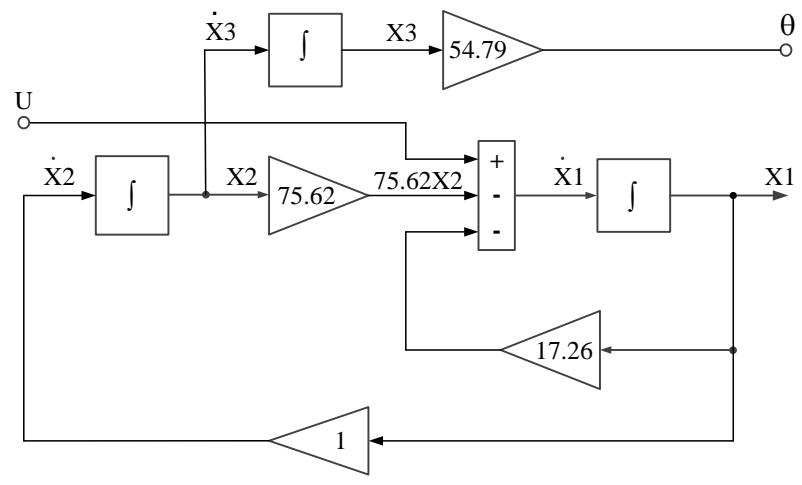

Figure 5 - Structural scheme of the robot arm link in the space of variables state

Proceeding from the condition of providing a given width of the zone within $\pm 2 \%$, a functional dependence between the system setup time and the values of the poles of the closed system was obtained in the following form:

$$
P_{p}=9.15 / T s \text {. }
$$

Having set the necessary values of $T s$ and determining with the help of the relation (32) the value of $P p$, the expanded matrices are written [19] taking into account the equations of the state of the object as follows: 


$$
\begin{aligned}
& A_{a}=\left[\begin{array}{ll}
A & 0 \\
C & 0
\end{array}\right], B_{a}=\left[\begin{array}{l}
B \\
0
\end{array}\right], K_{a}=\left[\begin{array}{ll}
K & K_{n+1}
\end{array}\right], \\
& A_{a s}=\left|\begin{array}{cccc}
-17.26 & -75.26 & 0 & 0 \\
1 & 0 & 0 & 0 \\
0 & 1 & 0 & 0 \\
0 & 0 & 54.79 & 0
\end{array}\right| \text {, } \\
& B_{a s}=\left|\begin{array}{l}
1 \\
0 \\
0 \\
0
\end{array}\right| \text {; } \\
& C_{a s}=\left|\begin{array}{lll}
0 & 0 & 54.79
\end{array}\right| .
\end{aligned}
$$

There is formed (Fig. 6) the block diagram of the servo drive of the control system of the robot arm link [20] in the feedback loop.

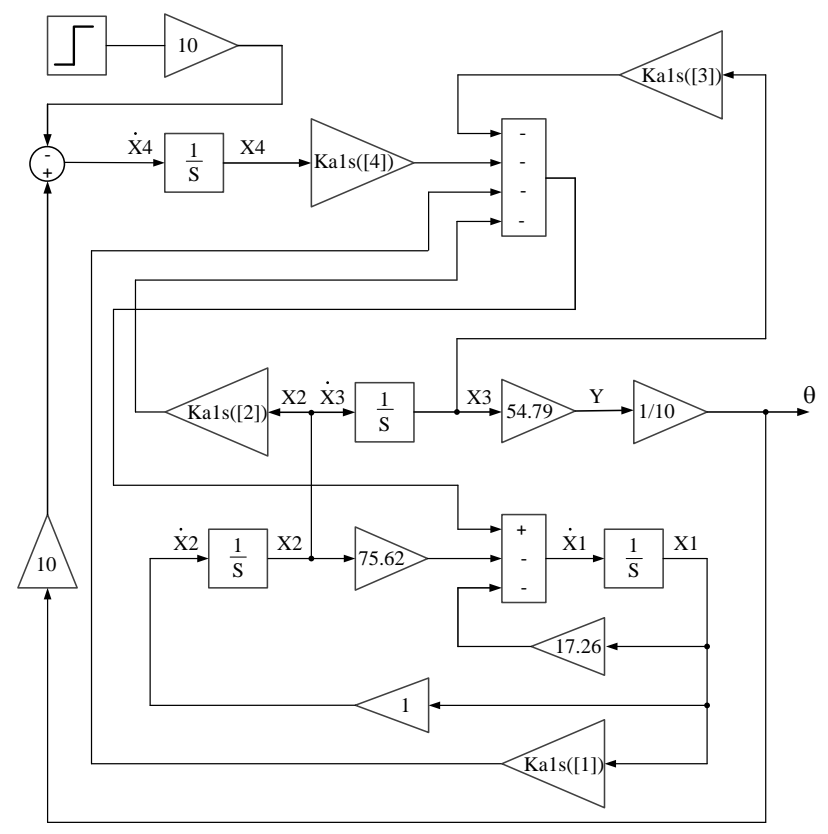

Figure 6 - The block diagram of the control system of servo-operated robot arm link in the feedback loop

The transfer function of the servo control system of the robot arm link in the feedback loop has the form as follows:

$$
W_{l}(s)=\frac{11.21}{s^{4}+7.23 s^{3}+20.09 s^{2}+24.51 s+11.21}
$$

In this case (Fig. 7), the coefficient $K_{3}$ can be connected in parallel to the series combination of integrator and linked with coefficient equal to $K_{n+1}$ in the direct path of the controller (in Fig.5 case Kals[3]), which will not change the characteristic equation of the system.

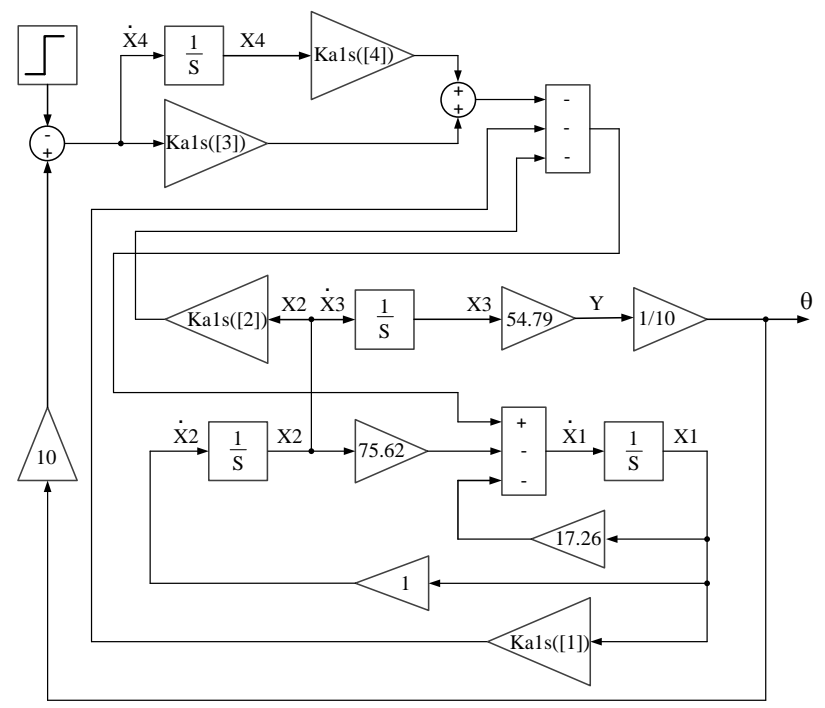

Figure 7 - Structural scheme of the control system of the servo-operated robot arm link in a direct path

\section{PROPOSED SYTHESIS METHOD DISCUSSION}

For nowadays, the synthesis of controller for a process in dynamic objects monitoring is based on the use of a root hodograph or frequency characteristics methods.

One of the peculiarities of the root hodograph method is that when used, the controller synthesis is performed in the time domain. It requires the use of a mathematical model of an object with characteristics that are close to the characteristics of a real object. In some cases it is difficult for implementation. In addition, the scope of the root hodograph method is limited due to the fact that it provides the possibility of location in a given area of the complex plane only one pair of poles and in cases where the order of the system is higher than the second, it is almost impossible to influence on the location of the other poles. Thus, if these poles in the complex plane occupy positions that do not allow to provide the specified dynamics of the system, then the synthesis of the controller based on the root hodograph method is reduced to the use of trial and error.

The controller synthesis using frequency characteristics is based on the use of the Nyquist stability criterion, which allows us to evaluate the stability of a closed system by its frequency characteristics when open. For the synthesis of the controller using frequency characteristics, the basic dynamic parameters of the system, in particular, the transmission coefficient of the open system, the margin of stability in the phase, or the frequency bandwidth, the time of installation, the efficiency of a disturbance compensation, etc., must be pre-set. When using frequency methods the insensitivity of a 
closed system characteristics to change its parameters can be to some extent ensured, which is essential for control systems with pronounced nonlinearities, the presence of which significantly complicates the process of controller synthesis. It should be noted that frequency methods involve the transition from the time domain of signal representation to the frequency with the subsequent procedure of controller synthesis, which significantly complicates the possibility of direct estimation of temporal characteristics of the system and, as a consequence, the choice of effective ways of their improvement.

In recent years, certain methods of synthesis of controllers, the implementation of which involves the possibility of placing all the poles of the transfer function of a closed system at specified points of a complex plane, have acquired certain development. The use of these methods requires the availability of information about all object state variables, measurement of which is not always possible due to the complexity of access to them, or the lack of appropriate measurement converters, estimates of data on variables that can be directly measured.

In order to accomplish the implementation task, it is necessary that the system model conforms to the canonical control form with characteristic equation (7). This requirement is difficult to fulfill because the state variables in such a model do not usually correspond to the state variables of the real system and therefore are not the values that should reflect the physical side of the processes occurring in the real system. In addition, in the general case, the state variables of the system, which is presented in the canonical form of control, may not be available for measurement. Using the Ackermann formula [17] based on the transformation of similarity translates a given model of an arbitrary structure into a canonical form of controllability, after which elements of the matrix $\mathrm{K}$ are determined by means of relations as follows:

$$
K_{i}=a_{i-1}-a_{i-1,} \quad i=1,2, \ldots n .
$$

The obtained solution is then converted back to its primary structure.

It should be noted that controlling the system state variables that are derived from the measurable state variables can cause considerable difficulty. Since the measured state variables values can vary in one direction, and the values of the calculated variables in the opposite direction. Therefore, if a calculated value is used to implement the control process, the state of the system then becomes inefficient, in particular, the system may become unstable, or it may significantly reduce the margin of safety.

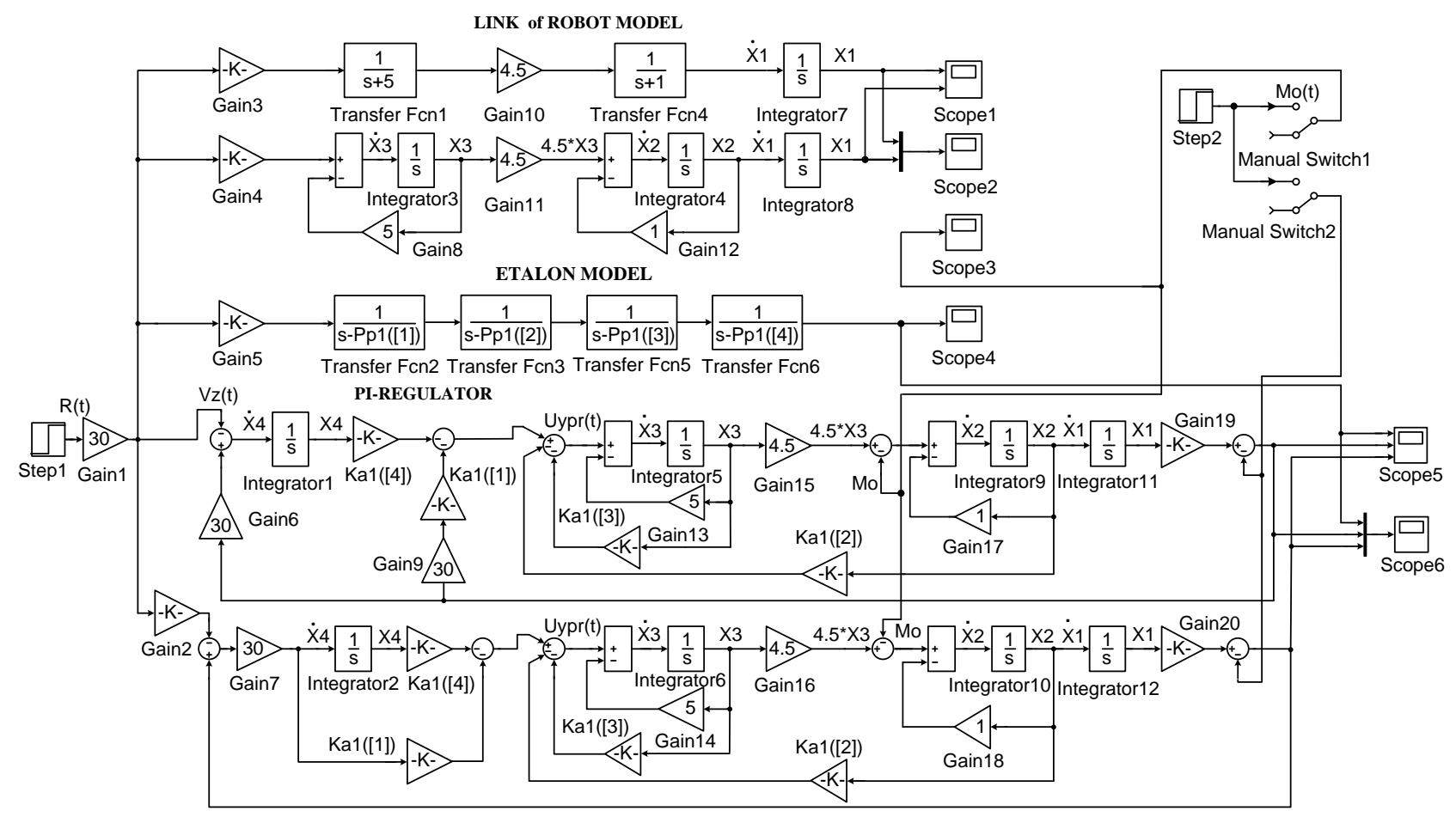

Figure 6 - Structural scheme of the control system of the servo-operated robot hand in the SIMULINK environment 


\section{CONTROL SYSTEM SIMULATIONS}

The block diagram of a control system of a robot hand servo drive in the SIMULINK environment [21-24] at the input step function acting, as well as when connecting to the input and output of the object of disturbing factors at the zero input signal is shown in Fig. 6. The environment covers the models of the robot's link both in the form of the transfer function and in the space of the state variables; the reference model and models of control system of the robot hand-arm of two types, namely, with the placing of the link with the coefficient Kals both in the circle of feedback, and in the direct path of the regulator. Transition functions for two implementations of the system with the inclusion of the link with coefficient $\mathrm{Kals}$ both in the feedback loop and in the direct path are shown in Fig. 7.

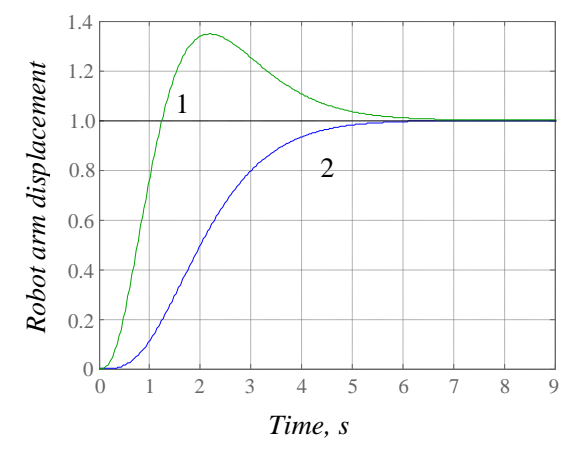

Figure 7 - Transitional characteristics of two implementations of the regulator by a servo-drive of an arm of a robot (curve 1 - the link with coefficient $K a l s$ is in the direct path; curve 2 - the link with coefficient $\mathrm{Kals}$ is in the feedback loop)

With the using transfer function $W_{2}(s)$, the reaction of the system is faster because the input signal in the form of a step function is directly brought to the input of the object (electric motor). In the case of the implementation of the transfer function $W_{l}(s)$, the step function is integrated; resulting in a sharp change in the input signal approximated by a linear function.

\section{CONCLUSIONS}

The peculiarities of constructing a mathematical model of one link of a robot's arm using MATLAB software package were considered. The synthesis of the regulator by a servo-drive of a robot arm was carried out by the method of placement of poles at given points of the complex plane. Synthesis was carried out for two implementations of the controller - with the inclusion of the link with coefficient
Kals both in the feedback loop circle and in the direct path of the system.

The simulation was carried out in SIMULINK environment at the input to the system of a single step (jump-like) function, as well as when connecting to the input and output of the object of disturbing factors at a zero signal at the input of the system. Both the implementation of the system, as in the introduction of $\mathrm{Kals}$ into the feedback loop and with the inclusion of Kals in a direct path, can be used in practice, but the first of them is appropriate to apply when it is not desirable to expose object of impact on the side of the jump effects of the input signal.

Due to the stated values of transition time and output signal displacement that provide the allowed divergence between output signal and sample proposed, the control system works in automatic mode.

\section{REFERENCES}

[1] F.C. Goodwin, S.F. Fraebe, M.E. Salgado, Control System Design, Prentice Hall, Upper Saddle River, New Jercey, 2001.

[2] R.C. Dorf, R.H. Bishop, Modern Control Systems, 13th Edition, Pearson Education, 2017.

[3] C.L. Phillips and R.D. Harbor, Feedback Control Systems, 3rd Edition, Prentice Hall, Upper Saddle River, New Jersey, 1996.

[4] S. Haykin, Neural Networks - A Comprehensive Foundation, Pearson Education, 2001.

[5] R.M. Cannon, Dynamics of Physical Systems, McGraw - Hill, New York, 1967.

[6] F.A. D'Souza, V.K. Garg, Advanced Dynamics, Prentice Hall, Englewood Cliffs, New Jersey, 1984.

[7] I.H. Shames, Engineering Mechanics: Statics and Dynamics, Prentice Hall, Englewood Cliffs, New Jersey, 1980.

[8] I.H. Shames, Introduction to Solid Mechanics, Prentice Hall, Englewood Cliffs, New Jersey, 1989.

[9] V.G. Krishnapura, A. Jutan, "ARMA neuron networks for modeling nonlinear dynamical systems," Canadian Journal of Chemical Engineering, vol. 75, pp. 574-582, 1997.

[10] T.W. Karjala, D.B. Himmelblau, "Data rectification for dynamic processes using artificial neural network," in: A.B.Bulsari (ed.), Computer Aided Chemical Engineering, vol. 6: Neural Networks for Chemical Engineers, Elsevier, Amsterdam, 1995, pp. 211-230.

[11] K.S. Narenda, K. Parthasarathy, "Identification and control of dynamical systems using neural 
networks," IEEE Transactions on Neural Networks, vol. 1, issue 1, pp. 4-27, 1990.

[12] Z. Lubosny, "Indentification of dynamic object by neural network," in: P.S. Szczepaniak (ed.). Computational Intellidence and Applications, Physica-Verlag, Heidelberg - New York, 1999, pp. 228-233.

[13] N. Bhat, T.J. McAvoy, "Use of neural nets for dynamic modeling and control of chemical process systems," Computers and Chemical Engineering, vol. 14, no. 4/5, pp. 573-582, 1990.

[14] D. H. Wolpert, K. Tumer, An Introduction to Collective Intelligence, NASA Ames Research Center, 2000.

[15] J.C. Hoskins, D.M. Himmelblau, "Artificial neural network models of knowledge representation in chemical engineering," Computers and Chemical Engineering, vol. 12, no. 9/10, pp. 81-890, 1988.

[16] G. Jinescu, V. Lavric, "The artificial neural networks and the drying process modeling," Drying Technology, vol. 13, no. 5-7, pp. 15791586, 1995.

[17] J.E. Ackermann, "Der Entwurf Linear Regelungs Systems in Zustandstraum," Regelungstech Process-Datenverarb, no. 7, pp. 297-300, 1972. (in German)

[18] M. Shahinpoor, A Robot Engineering Textbook, University of New Mexico Press, Albuquerque, 1984.

[19] G.H. Golub, C.F. Van Loan, Matrix Computations, John Hopkins University Press, Baltimore, 1989.

[20] K.S. Fu, R.C. Gonzalez, C.S.G. Lee, Robotics: Control, Sensing, Vision and Intelligence, McGraw-Hill Education (India) Pvt Limited, 1988.

[21] J. B. Dabney, T. L. Harmen, Mastering Simulink 4, Upper Saddle River, New Jersey: Prentice Hall, 2001.

[22] G.E. Forsyth, M.A. Malcolm, C.B. Moler, Computer Methods for Mathematical
Computation, Prentice Hall, Englewood Cliffs, New Jersey, 1977.

[23] E.O. Doebelin, Systems Modelling and Response, Wiley, New York, 1980.

[24] D.J. Edwins, Modal Testing: Theory and Practice, Research Studies Press distributed by Wiley, New York, 1984.

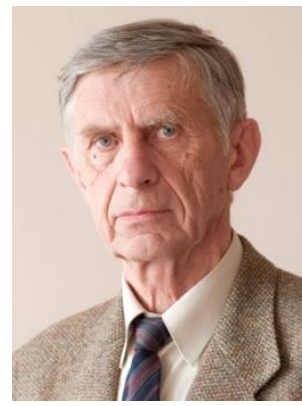

Markiyan Nakonechnyi is a Doctor of Technical Sciences, a professor at the Department of computerized automation systems at the Lviv Polytechnic National University, (Ukraine). M.Nakonechnyi is the author of more than 70 scientific works and copyright certificates. Areas of scientific interests: use of neural networks in automatic systems.

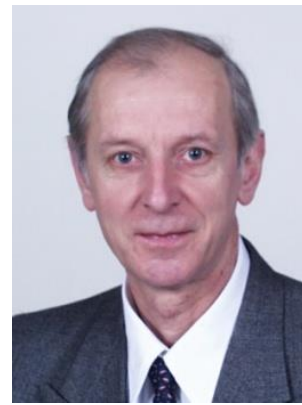

Orest Ivakhiv is a faculty member at the Computer Technology, Automation and Metrology Institute of Lviv Polytechnic National University. $\mathrm{He}$ has more than 300 publications, 12 patents. His research interests include electrical measurement and instrumentation, informative measurement theory, adaptive data processing, data compression, enumerative coding, communication theory and mechatronics.

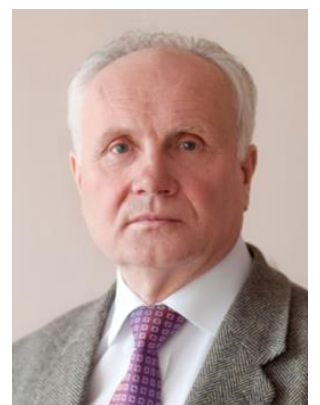

Oleksandr Viter is an Associate Professor of the Computerized Automation Systems Department of Lviv Polytechnic National University, (Ukraine). His scientific interests include automation and computerized information and measurement systems. $O$. Viter is the author of more than 70 scientific works and copyright certificates. 